\title{
miR-34b attenuates trauma-induced anxiety-like behavior by targeting CRHR1
}

\author{
JING ZHU $^{1 *}$, ZHEJUN CHEN $^{1 *}$, JINXING TIAN ${ }^{2}$, ZEHUI MENG ${ }^{1}$, \\ MINGDA JU $^{1}$, GENCHENG WU ${ }^{1}$ and ZHANZHUANG TIAN ${ }^{1}$

\begin{abstract}
${ }^{1}$ Department of Integrative Medicine and Neurobiology, State Key Laboratory of Medical Neurobiology, Collaborative Innovation Center for Brain Science, Institute of Acupuncture Research, WHO Collaborating Centre for Traditional Medicine, Fudan Institutes of Integrative Medicine, Fudan University, Shanghai 200032; ${ }^{2}$ Department of Anatomy, School of Basic Medicine, Shanghai University of Traditional Chinese Medicine, Shanghai 201203, P.R. China
\end{abstract}

Received January 5, 2016; Accepted April 25, 2017

DOI: $10.3892 / \mathrm{ijmm} .2017 .2981$

\begin{abstract}
Exposure to trauma is a potential contributor to anxiety; however, the molecular mechanisms responsible for trauma-induced anxiety require further clarification. In this study, in an aim to explore these mechanisms, we observed the changes in the hypothalamic pituitary adrenal (HPA) axis using a radioimmunoassay and the changes in anxiety-like behavior using the open field test and elevated plus maze test in a rat model following intervention with NBI-27914, a specific corticotropin-releasing hormone receptor 1 (CRHR1) antagonist. CRHR1 was found to be involved in trauma-induced anxiety. We then applied bioinformatic analysis to screen microRNAs (miRNAs or miRs) that target CRHR1, and miR-34b was determined to negatively regulate CRHR1 mRNA in primary hypothalamic neurons. The overexpression of miR-34b in the paraventricular nucleus (PVN) by a miRNA agomir using a drug delivery system decreased the hyperactivity of the HPA axis and anxiety-like behavior. Overall, the involvement of the HPA axis in trauma-induced anxiety was demonstrated, and trauma-induced anxiety was attenuated by decreasing the hyperactivity of the HPA axis via miR-34b by targeting CRHR1.
\end{abstract}

\section{Introduction}

Exposure to traumatic events is a risk factor for anxiety disorders (1). Acute stress-induced anxiety helps to maintain arousal and vigilance during dangerous conditions; however, anxiety

Correspondence to: Mr. Zhanzhuang Tian, Department of Integrative Medicine and Neurobiology, State Key Laboratory of Medical Neurobiology, Collaborative Innovation Center for Brain Science, Institute of Acupuncture Research, WHO Collaborating Centre for Traditional Medicine, Fudan University, 138 Yixueyuan Road, Xuhui, Shanghai 200032, P.R. China

E-mail:tianvv@shmu.edu.cn

*Contributed equally

Key words: miR-34b, corticotropin-releasing hormone receptor 1, anxiety, hypothalamic pituitary adrenal axis is unfavorable for the recovery of a subset of patients that have experienced emergency major surgery or trauma $(2,3)$. Anxiety emerges sometimes in the aftermath of major surgery or incident injury. This has been increasingly established in traumatic events research, which is a growing area of focus (3-5).

Several approaches have been used to solve this issue, including psychotherapy and anti-depressant medications (6), with little success. Furthermore, the application of certain medications during the peri-operative period sometimes produces undesired reactions (7), such as a risk for bleeding (8). The dysfunction of the hypothalamic pituitary adrenal (HPA) axis is one of the most widely accepted hypothesis of anxiety (9), which is a central system in maintaining neuroendocrine equilibrium.

The HPA axis is composed of corticotropin-releasing hormone $(\mathrm{CRH})$ that is secreted from the hypothalamus and successively stimulates adrenocorticotropic hormone (ACTH) secretion from the pituitary gland. Glucocorticoid hormones [human, cortisol; rodent, corticosterone (CORT)] are then secreted from the cortex of the adrenal glands. The HPA axis has an established association with anxiety, including physiological homeostasis and the stress response $(10,11)$. Disturbances in this system result in severe hormonal imbalances, which may be strongly implicated in the pathology of major depressive disorder (9); however, the potential role of the HPA axis in trauma-induced anxiety remains unknown.

MicroRNAs (miRNAs or miRs) are small, non-coding RNAs that typically bind to specific sequences in the 3'-untranslated regions (3'-UTRs) of targeted mRNAs to negatively fine-tune protein expression. miRNAs have recently emerged as a key regulator of metabolism and many are considered to be biomarkers of depression or anxiety (12). miRNAs play an important role in mediating anxiety via glucocorticoid (GC) hormone signaling. There is evidence that miR-124a may mediate anxiety via GC/glucocorticoid hormone receptor (GR) signaling (13) and that miR-608 may affect anxiety and CORT (14); however, the molecular mechanisms through which major surgery induces anxiety and the involvement of miRNAs in the hypothalamus in mediating the HPA axis require further investigation.

This study aimed to investigate the role of the HPA axis in trauma-induced, anxiety-like behavior and to evaluate whether miRNAs in the hypothalamus are involved in a rodent model. 


\section{Materials and methods}

Animals. All animal experiments performed on rats were conducted in accordance with NIH Guidelines (NIH Publications no. 8023, revised in 1978) and approved by the Animal Use and Care Committee of Fudan University, Shanghai, China. Adult male Sprague-Dawley (SD) rats $(\mathrm{n}=51$; weighing, 200 $\pm 20 \mathrm{~g})$ and 1-day-old neonatal SD rats $(n=100)$ were purchased from the Experimental Animal Center of the Chinese Academy of Sciences (Shanghai, China) and housed in a quiet room with a 12:12 light/dark cycle with ad libitum access to food and water. Room temperature was maintained at $25 \pm 2^{\circ} \mathrm{C}$.

Surgery. The adult male Sprague-Dawley rats in the model group were subjected to hepatectomies under anesthesia by sodium pentobarbital $(30 \mathrm{mg} / \mathrm{kg})$. Briefly, an approximately 7-cm-long surgical incision was made from the xiphoid process to the pubic symphysis along the abdomen. From this incision, $10 \%$ of the liver was removed from the right lobe. The incision was then closed following exhaustive hemostasis. All rats were kept warm under a $22^{\circ} \mathrm{C}$ environment during the hepatectomy and covered with a sterile gauze after hepatectomy. All surgeries were performed between 8:00 and 10:00 a.m. The rats in the sham-operated (sham) group were administered only anesthesia and only an incision was made. No intervention process was performed on the rats in the intact group.

Animal groups. In the first set of animal experiments the SD rats were divided into the intact, sham and model group. There were 7 rats in each group. In the second set of animal experiments, the SD rats were divided into the hepatectomy + vehicle and hepatectomy + NBI-27914 group. There were 7 rats in each group. In the third set of animal experiments, the SD rats were divided into the miR-NC-A, miR-34b-A, hepatectomy + miR-NC-A and hepatectomy + miR-34b-A group. There were 4 rats in each group.

Tissue collection. The rats in each group were sacrificed by decapitation. Their brains were immediately removed and the hypothalamus was separated from the brain. All samples were snap-frozen in liquid nitrogen and then stored at $-80^{\circ} \mathrm{C}$ until further processing.

Radioimmunoassay (RIA). Blood samples were collected by decapitation at the time of sacrifice and the serum was separated by centrifugation. The concentrations of $\mathrm{CRH}, \mathrm{ACTH}$ and CORT were determined by RIA kits that were purchased from the Beijing Sinouk Institute of Biological Technology (Beijing, China). All the samples were assayed together, with each sample analyzed in duplicate.

Cell culture. Primary cultures of fetal hypothalamic neuronal cells were prepared from 1-day-old neonatal Sprague-Dawley rats, as previously described (15). In brief, their brains were immediately removed, and the hypothalami were separated and placed in ice-cold Hanks' balanced salt solution (HBSS, 14065056; Gibco Waltham, MA, USA). The hypothalamic tissue was placed in $0.125 \%$ pancreatic enzymes (25200056; Gibco) for $20 \mathrm{~min}$ after being removed from the vessel. The hypothalamic cells were harvested by centrifugation $(1,000 \mathrm{rpm}, 5 \mathrm{~min}$, $4^{\circ} \mathrm{C}$ ) following filtration using a strainer (352350; BD Falcon, Franklin Lakes, NJ, USA). The cells were resuspended by neurobasal medium (21103049; Gibco) that was supplemented with $10 \%$ fetal bovine serum (FBS; 12657-029; Gibco) and plated on culture plates (353047; BD Falcon) at approximately 16,000 cells/ $\mathrm{cm}^{2}$ and then incubated at $37^{\circ} \mathrm{C}$ in an atmosphere of $95 \%$ air, $5 \% \mathrm{CO}_{2}$ overnight. We changed the culture solution to neurobasal medium that contained 1\% B27 (17504-044; Life Technologies, Waltham, MA, USA) the following day and we then changed half the medium every 3 days. The cells were maintained for 1 week with this medium before experimental use.

293T cells (http://www.cellbank.com.cn/) were grown in Dulbecco's modified Eagle's medium (DMEM; 12491-015; Invitrogen, Carlsbad, CA, USA) containing 10\% FBS. The cells were also incubated at $37^{\circ} \mathrm{C}$ in a humidified atmosphere of $5 \% \mathrm{CO}_{2}$.

Pharmacological applications and miRNA transfection. Drugs were injected into the paraventricular nucleus (PVN) in vivo according to the rat brain in stereotaxic coordinates third at AP $1.5 \mathrm{~mm}$, DV $0.4 \mathrm{~mm}, \mathrm{H} 7.8 \mathrm{~mm}$ using a drug delivery system from RWD Life Science, Shenzhen, China (RWD, 62037, 62137, 62237). This system was fixed into the rat brain 10 days before an experiment to avoid unnecessary disturbances. The rats were administered injections of the vehicle $(0.5 \mu 10.9 \%$ saline $)$ or 5-chloro-4-[ $N$-(cyclo-propyl) methyl- $N$-propylamino]-2-m ethyl-6-(2,4,6-trichlor-ophenyl) amino-pyridine (NBI-27914: 5 nmol; 184241-44-9; Sigma, St. Louis, MO, USA) which was dissolved in the vehicle before the surgery.

miRNA mimics for miR-351, miR-34b, miR-34c, miR-24, miR-204, miR-214, miR-27a, miR-122, miR-150, miR-216a and miR-218, agomirs (miR--34b) and their negative controls (miR04201-1-10) were commercially synthesized from RuiBio, Guangzhou, China. These miRNA mimics and control were transfected into the hypothalamic neurons and 293T cells using transfection reagent (FuGENE ${ }^{\circledR}$ HD Transfection Reagent, E2312; Promega Madison, WI, USA), according to the manufacturer' $\mathrm{s}$ instructions in vitro. miR-34b agomir and its negative control were injected into the PVN using the drug delivery system at 1 week and 3 days before the experiment, each at a concentration of $5 \mathrm{nmol}$.

Following transfection, the hypothalamics neurons from primary culture were incubated with the vehicle or $10 \mu \mathrm{M}$ forskolin, and these cells were harvested at 2, 4 and $24 \mathrm{~h}$ after the forskolin application.

Open field test (OFT) and elevated plus maze test. One day after surgery, all the rats were transferred to a quiet room to assess their anxiety activity. Each rat was placed in the center of a black polycarbonate box (100x100x48 $\mathrm{cm}$, length $\mathrm{x}$ width $\mathrm{x}$ height), which had been cleared by $75 \%$ ethanol to reduce the inference of odors. Their locomotor activity, times of crossing the central area and forelegs lifting from the floor were recorded for $5 \mathrm{~min}$ using a video camera that was mounted $100 \mathrm{~cm}$ above the arena.

One hour after OFT, the anxiety-like behavior of the rats was determined using the elevated plus maze (EPM) test. It was a cross-shaped platform constituted of 4 arms $(50 \times 10 \mathrm{~cm})$ and was $50 \mathrm{~cm}$ elevated above ground. The rats were placed in the central area $(10 \times 10 \mathrm{~cm})$ of the maze cleared by $75 \%$ ethanol towards an open arm. Their behavior were recorded for $5 \mathrm{~min}$ using a video camera that was mounted $100 \mathrm{~cm}$ above the arena.All data 
were analyzed using Xinruan software (XR-Xmaze+; Xinruan, Shanghai, China).

Real-time-polymerase chain reaction $(R T-P C R)$. For the analysis of mRNA expression, total hypothalamic RNA was extracted using TRIzol reagent (15596-026; Invitrogen) according to the manufacturer' $\mathrm{s}$ instructions. The purity and integrity of the RNA were examined spectroscopically before obtaining cDNA using the GoScript ${ }^{\mathrm{TM}}$ Reverse Transcription system (M1705; Promega). The reactions were set up with $10 \mu \mathrm{l}$ SYBR-Green Real Master Mix (Promega), $1.6 \mu \mathrm{l}$ primer mixture $(200 \mathrm{nM})$, and $1.6 \mu \mathrm{l} \mathrm{cDNA}$ template. The thermal cycling conditions were as follows: $95^{\circ} \mathrm{C}, 3 \mathrm{~min}$ for denaturation, followed by 38 cycles of $95^{\circ} \mathrm{C}, 10 \mathrm{sec}$, and $60^{\circ} \mathrm{C}, 30 \mathrm{sec}$, and $72^{\circ} \mathrm{C}$ for $30 \mathrm{sec}$. After the cycles, a melting curve analysis was performed to ensure the purity of PCR products.

For the analysis of miRNA expression, miRNA was extracted using the miRcute miRNA assay (DP501; Tiangen Biotech, Beijing, China). The purity and integrity of the RNA were also examined spectroscopically before obtaining cDNA using the GoScript $^{\mathrm{TM}}$ Reverse Transcription system (M1705; Promega).

The primers that were used for the analysis of mRNA and miRNA expression (Table I) were designed and synthesized by Invitrogen and purified by high performance liquid chromatography (HPLC). All experiments were run in triplicate and relative mRNA and miRNA levels were analyzed by means of the $2^{-\Delta \Delta \mathrm{Ct}}$ method and normalized to glyceraldehyde 3-phosphate dehydrogenase (GAPDH) and U6 RNA, respectively.

Western blot analysis. The hypothalamic tissue was isolated from the rat brains and homogenized in RIPA buffer (9806; Cell Signaling Technology, Danvers, MA, USA). Following calibration by the BCA protein assay kit (23225; Pierce Pharmaceuticals, Waltham, MA, USA) the hypothalamic supernatant was denatured for $10 \mathrm{~min}$ at $100^{\circ} \mathrm{C}$ in a solution of $4 \mathrm{X}$ Laemmli sample buffer (161-0747; Bio-Rad, Hercules, CA, USA). The proteins were separated using Bio-Rad equipment (PowerPac Universal; Bio-Rad) and transferred onto polyvinylidene fluoride (PVDF) membranes (ISEQ00010; Merck Millipore, Darmstadt, Germany). The PVDF membranes were then incubated in $5 \%$ non-fat milk for $1 \mathrm{~h}$ at room temperature and incubated at $4^{\circ} \mathrm{C}$ in primary antibodies overnight (CRHR1: AP01194PU-N, 1:500; Acris Antibodies GmbH, Herford, Germany).

After washing in buffer (TBS-0.1\% Tween-20), the membranes were incubated with HRP-conjugated rabbit anti-goat IgG (H+L) (SA00001-4; Proteintech, Chicago, IL, USA), diluted at 1:10,000, for $2 \mathrm{~h}$ at $4^{\circ} \mathrm{C}$. Target protein signals were detected using an ECL detection kit (Immobilon Western Chemiluminescent Horseradish Peroxidase Substrate p90720; Millipore) and exposed using an Image Quant LAS 4000 mini (GE Healthcare, Buckinghamshire, UK). The signals were quantified using Quantity One software. The results for signal intensity were expressed in arbitrary densitometric units, after normalizing to GAPDH (ab181602; Abcam, Cambridge, UK) as an internal standard.

Immunofluorescence (IF). After being cultured for 1 week, the cells were washed with hanks balanced salt solution (HBSS: C0218; Beyotime, Jiangsu, China) and fixed using 4\% paraformaldehyde (PFA) in $0.01 \mathrm{M}$ phosphate-buffered saline (PBS)
Table I. Primers for RT-PCR.

Gene

symbol

Primer sequences

CRH F: CTC TCT GGA TCT CAC CTT CCA C

R: CTA AAT GCA GAA TCG TTT TGG C

CRHR1 F: TGG AAC CTC ATC TCG GCT TT R: GTG AGC TGG ACC ACA AAC CA

CRHR2 F: TTC CTG CTG CAA CTC ATC GA R: GCG GCA CCA GAC CTC ATT

AVP F: TGC CTG CTA CTT CCA GAA CTG C R: AGG GGA GAC ACT GTC TCA GCT C

AVPR1a F: GCG GAA AGA CAG CGT CCT CGC GAC A R: GCT CAT GCT ATC GGA GTC ATC CTT GGC GAA T

AVPR1b F: AGA TTC TAC CAA TGT GGC TTT C R: ATG GTG GCT CAA GGA ACG

rno- $\quad$ F: ACA CTC CAG CTG GGA GGC AGT GTA ATT AGC miR-34b R: TGG TGT CGT GGA GTC G

U6 F: CTC GCT TCG GCA GCA CA R: AAC GCT TCA CGA ATT TGC GT

GAPDH F: GTA TGA CTC TAC CCA CGG CAA GT R: TTC CCG TTG ATG ACC AGC TT

F, forward; R, reverse; CRH, corticotropin-releasing hormone; CRHR, corticotropin-releasing hormone receptor; AVP, arginine vasopressin; AVPR1a, arginine vasopressin receptor $1 \mathrm{~A}$.

at room temperature for $10 \mathrm{~min}$. They were then blocked with $10 \% \mathrm{FBS}$ at $37^{\circ} \mathrm{C}$ for $1 \mathrm{~h}$ before being incubated in a mouse polyclonal antibody against NeuN (ABN78; 1:1,000; Millipore) diluted in PBS containing $1 \%$ bovine serum albumin (BSA: 9048-46-8; Amersco, Solon, OH, USA), 0.02\% sodium azide and $0.03 \%$ Triton $\mathrm{X}-100$ at $4^{\circ} \mathrm{C}$ overnight. After rinsing, the cells were incubated in secondary antibody solutions [1:500, Alexa Flour 488 donkey anti-mouse $\operatorname{IgG}(\mathrm{H}+\mathrm{L})$ antibody, A11032; Life Technologies] for $1 \mathrm{~h}$ at room temperature. The cells were analyzed using a Fluorescence microscope (Leica, Wetzlar, Germany).

Luciferase reporter assay. A forward primer (GCTAGCTGC AAGCTCACTGACGAGCC) and reverse primer (TCTAGAG GACTGGACCATTCTAACCC) were used to amplify the segment of CRHR1 3'-UTR sequence by RT-PCR. The Dual-Luciferase reporter genes were constructed using the psiCHECKTM-2 vector (Promega) and the 3'-UTR sequences of rat CRHR1. The sequences were introduced between the NotI and XhoI sites to Renilla luciferase 3'-UTR. The Firefly luciferase vector was used for internal reference. Constructs with mutated 3'-UTR of CRHR1 were used as negative controls.

The 293T cells were cultured in DMEM that was supplemented with $10 \%$ FBS. A total of $4 \times 10^{4}$ cells/well were seeded onto 24 -well plates. Following $24 \mathrm{~h}$ in culture, the cells were transfected using transfection reagent $\left(\mathrm{FuGENE}^{\circledR} \mathrm{HD}\right.$ Transfection Reagent, E2312; Promega) with a mixture containing $200 \mathrm{ng} / \mathrm{ml}$ of the Dual-Luciferase reporter plasmid and $40 \mathrm{nM}$ miR-34b mimic or NC mimic. The cells transfected with the vectors containing a mutation in the 3 '-UTR 

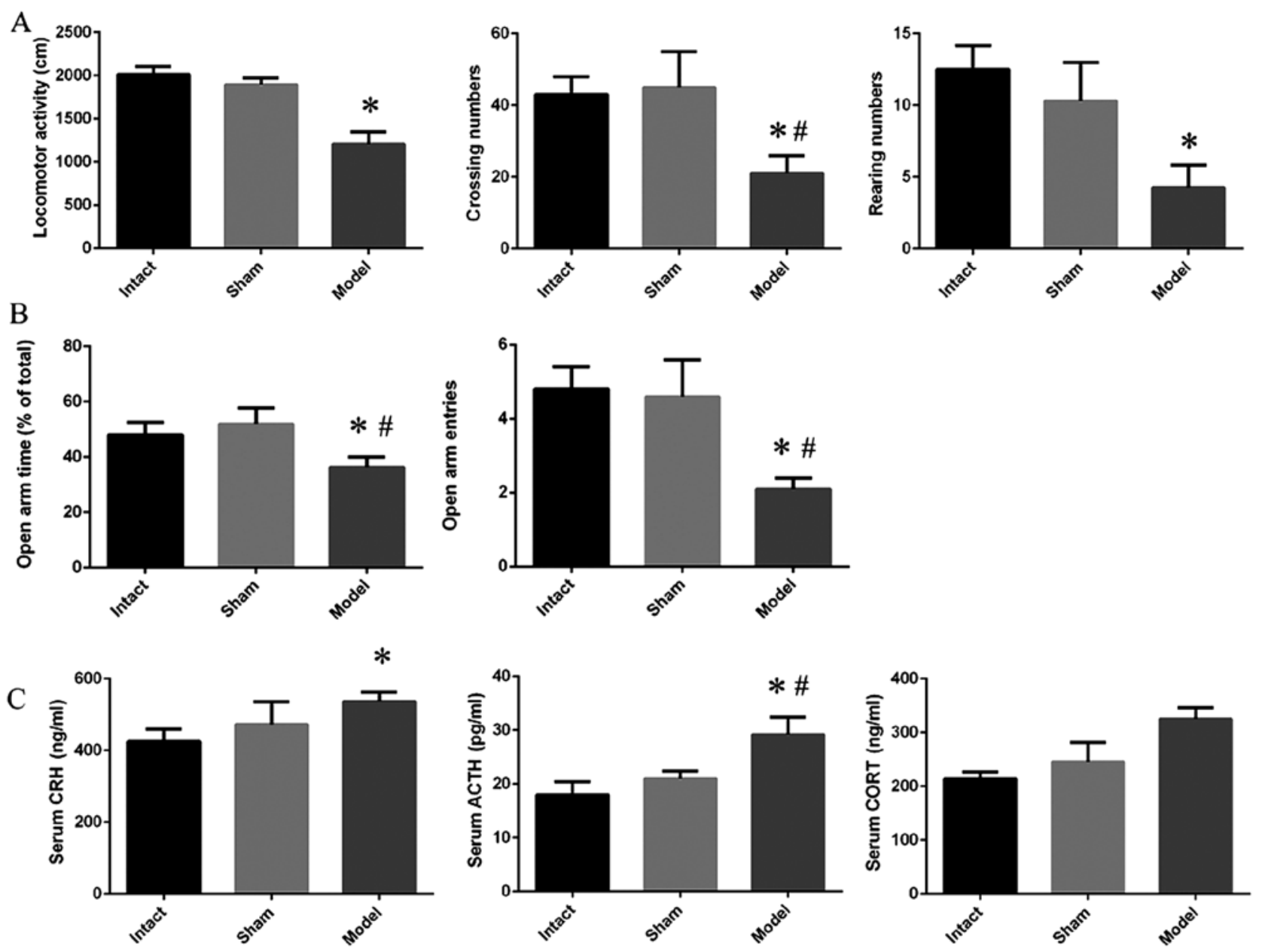

D
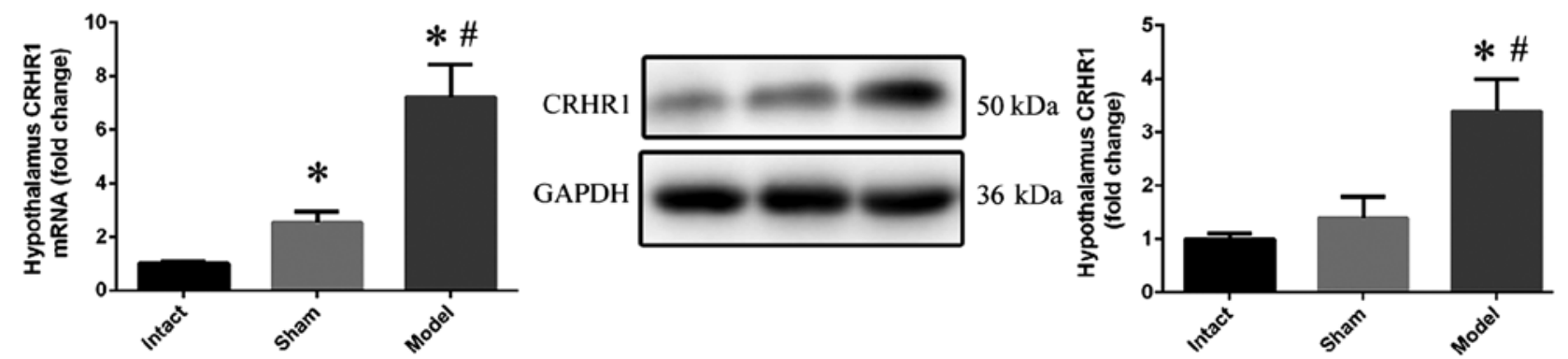

Figure 1. Surgery-induced anxiety and hyperactivity of the hypothalamic pituitary adrenal (HPA) axis. (A) Rat performance in an open field test after surgery. (B) Rat performance in elevated plus maze test after surgery. (C) $\mathrm{CRH}$, adrenocorticotropic hormone (ACTH) and CORT levels in serum after surgery detected by radioimmunoassay. (D) Representative western blots of corticotropin-releasing hormone receptor 1 (CRHR1) are shown with the quantification of mRNA and protein levels in the hypothalamus after surgery; $n=7$ for each group. ${ }^{*} p<0.05$ represents statistically significant differences when compared with the intact group; ${ }^{p} \mathrm{p}<0.05$ represents statistically significant differences compared with the sham-operated (sham) group.

of CRHR1 (p-Luc-3_-UTR MUT CRHR1) served as controls for normalization. When the cells were transfected for $24 \mathrm{~h}$, the luciferase activity was measured using a Dual-Lucferase ${ }^{\circledR}$ Reporter assay system (E1910; Promega). All transfections were repeated independently 3 times.

Statistical analysis. The data are presented as the mean \pm standard deviation (SD) and were analyzed using SPSS 17.0 software. For statistical comparisons, the values were subjected to a one-way ANOVA followed by Tukey's test among the groups. A P-value of $<0.05$ was considered to indicate a statistically significant difference.

\section{Results}

Surgery-induced anxiety-like behavior and hyperactivity of the HPA axis. The results of the OFT revealed a significant decrease in locomotor activity in the model group compared with the intact group $(\mathrm{p}<0.05)$. Moreover, the crossing numbers $(\mathrm{p}<0.05)$ and rearing numbers $(\mathrm{p}<0.01)$ in the model group also exhibited a statistically significant decreasing trend when compared with the intact group (Fig. 1A). In the EPM test, the rats in the model group demonstrated a lower open arm time $(p<0.05)$ and open arm entries $(p<0.01)$ compared with those in the intact and sham group (Fig. 1B). Moreover, the concentrations of serum 
A
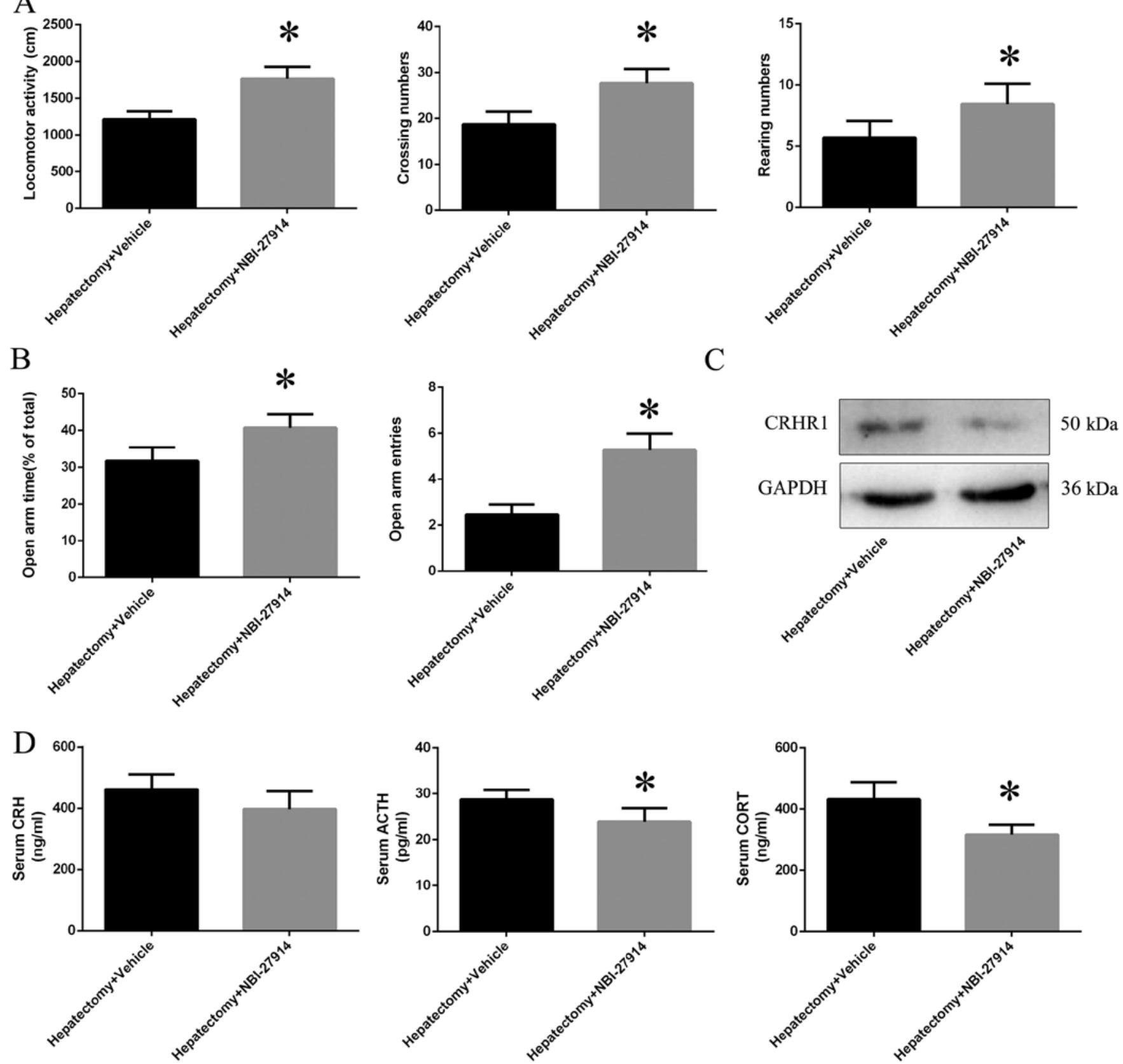

Figure 2. Surgery-induced anxiety and hyperactivity of the hypothalamic pituitary adrenal (HPA) axis following treatment with NBI-27914. (A) Rat performance in open field test. (B) Rat performance in elevated plus maze test. (C) Representative western blots of the hypothalamus CRHR1 are shown following treatment with NBI-27914. (D) CRH, adrenocorticotropic hormone (ACTH) and CORT levels in serum; $n=7$ for each group. "p $<0.05$ represents a statistically significant difference when compared with the hepatectomy + vehicle group.

CRH $(p<0.05)$, ACTH $(p<0.01)$ and CORT $(p<0.001)$ increased significantly in the model group when compared with the intact group (Fig. 1C). In addition, the mRNA $(\mathrm{p}<0.001)$ and protein $(\mathrm{p}<0.01)$ expression of CRHR1 in the model group was upregulated when compared with the intact group (Fig. 1D).

Surgery-induced anxiety and hyperactivity of the HPA axis is blocked by NBI-27914. Increases in locomotor activity $(\mathrm{p}<0.01)$, crossing numbers $(\mathrm{p}<0.05)$ and rearing numbers $(\mathrm{p}<0.01)$ in the OFT were observed in the hepatectomy + NBI-27914 group when compared with the hepatectomy + vehicle group (Fig. 2A); we also observed increases in both the open arm time $(\mathrm{p}<0.05)$ and entries $(\mathrm{p}<0.01)($ Fig. $2 \mathrm{~B})$. Moreover,
CRHR1 protein expression level was downregulated in the hepatectomy + NBI-27914 group $(\mathrm{p}<0.01)$ compared with the hepatectomy + vehicle group (Fig. 2C). The concentrations of serum ACTH $(\mathrm{p}<0.05)$ and CORT $(\mathrm{p}<0.01)$ were decreased in the hepatectomy + NBI-27914 group when compared with the hepatectomy + vehicle group; however, there was no difference in the levels of CRH ( $>0.05)$ between the hepatectomy + vehicle and hepatectomy + NBI-27914 group (Fig. 2D).

Forskolin induces $C R H$ and AVP signaling in primary hypothalamic neurons. After 7 days in culture, hypothalamic neuronal cells were observed by NeuN using immunofluorescence, showing that approximately $90 \%$ of 

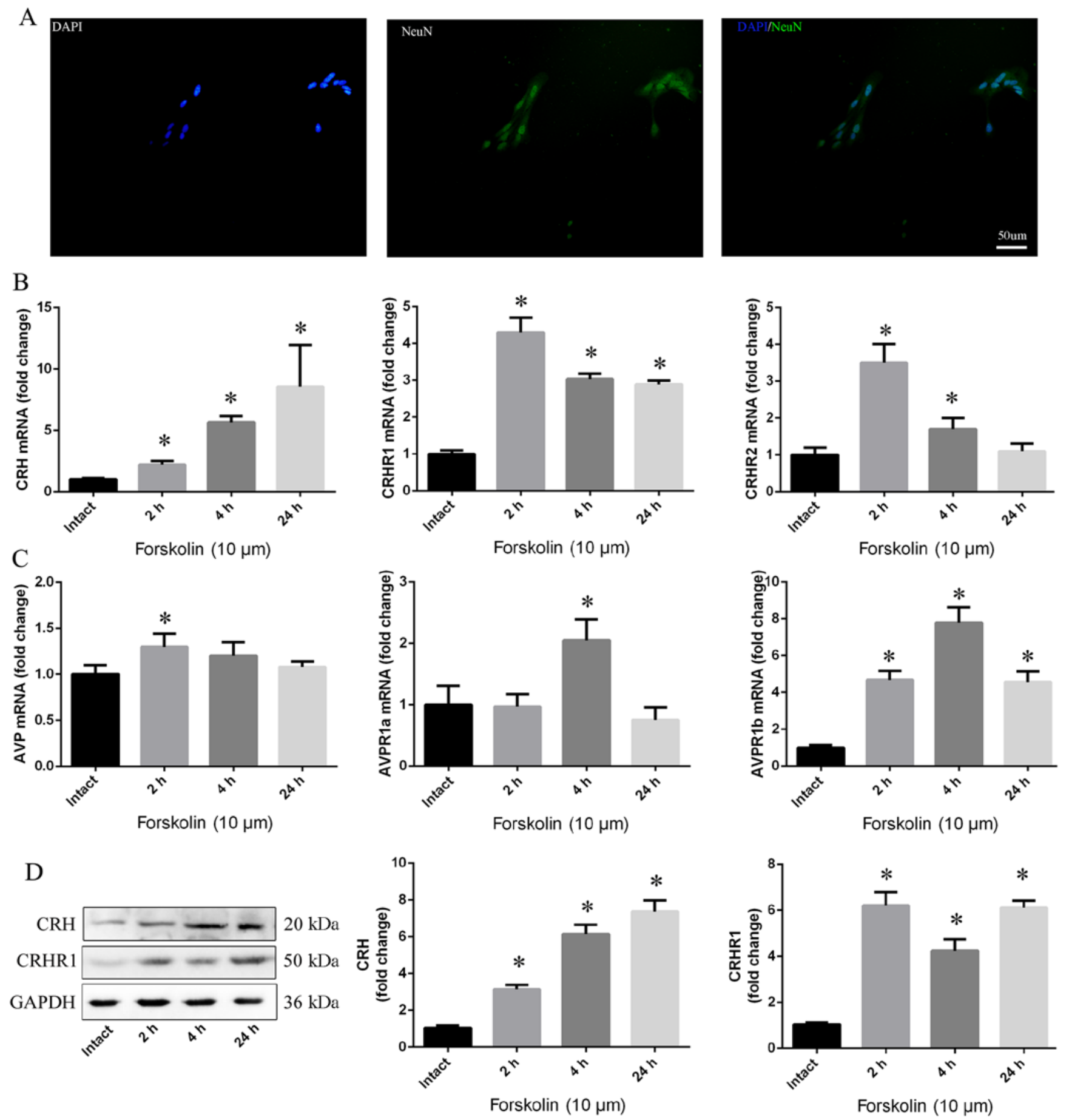

Figure 3. Corticotropin-releasing hormone (CRH) and CRHR1 induced by forskolin (10 $\mu \mathrm{m})$ in primary cultured hypothalamus neurons. (A) Primary hypothalamic neurons were examined using NeuN antibody by immunofluorescence. (B) Quantification of CRH, CRHR1 and CRHR2 mRNA levels in the primary cultured hypothalamic neurons. (C) Quantification of AVP, AVPR1a and AVPR1b mRNA level in the primary cultured hypothalamic neurons. (D) Representative western blots of $\mathrm{CRH}$ and $\mathrm{CRHR} 1$ are shown with quantification data in primary cultured hypothalamic neurons; $\mathrm{n}=4$ for each group. * $\mathrm{p}<0.05$ represents a statistically significant difference compared with the intact group.

the cells were neurons (Fig. 3A). The mRNA levels of CRH, CRHR1, CRHR2, AVP, AVPR1a and AVPR1b were enhanced in response to forskolin, which was observed after 2, 4 and $24 \mathrm{~h}(\mathrm{p}<0.05)$ (Fig. 3B and C). Moreover, a similar trend in $\mathrm{CRH}$ and CRHR1 protein levels was observed in the hypothalamic neurons $(\mathrm{p}<0.05)$ (Fig. 3D).

CRHRl is a target of miR-34b. From previous bioinformatic analyses, particularly research from http://www.targetscan.org/ vert_61/ and http://zmf.umm.uni-heidelberg.de/apps/zmf/ mirwalk2/, miR-24, miR-34b, miR-34c, miR-27a, miR-122, miR-128, miR-150, miR-204, miR-214 and miR-216a were identified as candidate miRNAs for binding to the 3'-UTR of CRHR1 mRNA (Fig. 4A). Following the overexpression of these miRNAs, CRHR1 mRNA was found to be decreased following the administration of miR-27a $(\mathrm{p}<0.05)$ and miR-34b (p<0.05) (Fig. 4B). miR-34b was observed to be decreased in response to forskolin whithin 1 day $(p<0.05)$ 
A

B
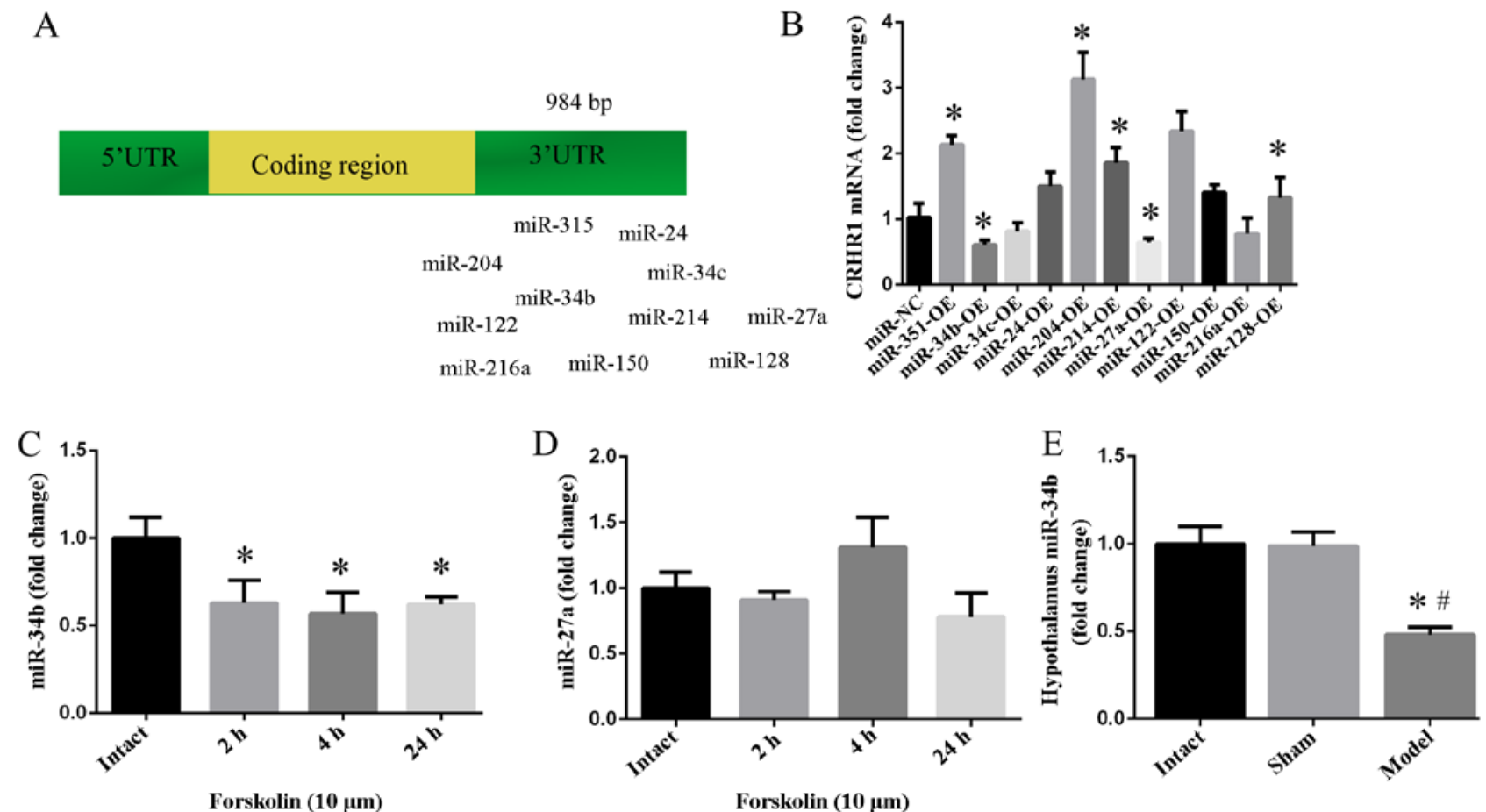

Figure 4. Screening of miRNAs that regulate 3'-untranslated region (3'-UTR) activity of corticotropin-releasing hormone receptor 1 (CRHR1) mRNA in hypothalamus neurons. (A) Screening of miRNAs that regulate the 3'-UTR activity of CRHR1 mRNA, as determined by bioinformatics. (B) CRHR1 mRNA level after overexpression treatment with each miRNA in primary cultured hypothalamus neurons. (C) Expression of miR-34b after treatment by forskolin in primary cultured hypothalamus neurons. (D) Expression of miR-27a after treatment with forskolin in primary cultured hypothalamus neurons. (E) Hypothalamus miR-34b level in rats after surgery. $n=4$ for each group in (B-D) while $n=7$ in $(E)$. $p<0.05$ represents a statistically significant difference compared with the intact group or miRNA-NC group; ${ }^{*} \mathrm{p}<0.05$ represents statistically significant difference compared with the sham group.

(Fig. 4C), while there was no significant effect on miR-27a (p>0.05) (Fig. 4D). Compared with the intact group, hypothalamic miR-34b in the model group exhibited a decreasing trend $(\mathrm{p}<0.01)$ (Fig. 4E).

The genes with a mutation in the seed region of the 3'-UTR of CRHR1 were unable to bind to miR-34b (Fig. 5A), thereby not eliminating CRHR1 mRNA. The luciferase activity of the cells transfected with miR-34b expression and p-Luc-3'-UTR CRHR1 was decreased by $50 \%$ when compared with the cells that were co-transfected with miR-NC mimic and p-Luc-3'-UTR CRHR1 $(\mathrm{p}<0.05)$. The negative control construct of mutations in the 3'-UTR of CRHR1 showed no obvious change in luciferase activity ( $p>0.05)$ (Fig. 5B). The overexpression of miR-34b downregulated the CRHR1 protein levels $(\mathrm{p}<0.05)$ (Fig. 5C). Moreover, there was an increase in the CRHR1 mRNA level by forskolin in the primary hypothalamus after 1 day $(\mathrm{p}<0.01)$, while the overexpression of miR-34b attenuated this increase in the CRHR1 mRNA level compared with the miR-NC group $(\mathrm{p}<0.01)$ (Fig. 5D).

Surgery-induced anxiety and hyperactivity of the HPA axis is blocked by the overexpression of miR-34b. Using cerebral stereotaxis, miR-34b agomiR was used to induce the overexpression of miR-34b in the hypothalamus before surgery. As shown by the results of OFT, the locomotor activity $(\mathrm{p}<0.01)$ was decreased in the hepatectomy + miR-NC-A group compared with the miR-NC-A group. The overexpression of miR-34b using agomir in the rats in the hepatectomy + miR-34b-A group increased their locomotor activity $(\mathrm{p}<0.01)$ compared with the rats in the hepatectomy + miR-NC-A group (Fig. 6A). Furthermore, both the open arm time $(p<0.01)$ and entries $(p<0.05)$ were decreased in the hepatectomy + miR-NC-A group compared with the miR-NC-A group, and the open arm time was increased $(p<0.05)$ in the hepactomy + miR-34b-A group compared with the hepatectomy + miR-NC-A group (Fig. 6B). In addition, we found that CRHR1 expression in the hepatectomy + miR-NC-A group was increased compared with the miR-NC-A group ( $\mathrm{p}<0.001)$, and was decreased in the hepatectomy + miR-NC-A group $(\mathrm{p}<0.05)$ compared with the hepatectomy + miR-34b-A group (Fig. 6C).

The ACTH $(\mathrm{p}<0.05)$ and CORT $(\mathrm{p}<0.01)$ levels were upregulated in the hepatectomy + miR-NC-A group when compared with the miR-NC-A group. Moreover, the levels of ACTH $(p<0.05)$, CORT $(p<0.05)$ in the hepatectomy + miR-34b-A group were decreased when compared with those in the hepatectomy + miR-NC-A (Fig. 6D).

\section{Discussion}

Trauma affects a large number of individuals and must be considered a significant public health issue. Trauma-related disorders, particularly anxiety, are commonly experienced by patients who are exposed to emergency trauma or undergo surgery. Patients with post-surgery depression have been found to be a host of poor surgical recovery outcomes (2).

Anxiety is often characterized by a malfunction of the HPA axis (16), which is a hormonal pathway that is initiated by the release of CRH from the PVN in the hypothalamus. Thus, the release of CRH stimulates the secretion of ACTH into the 
A

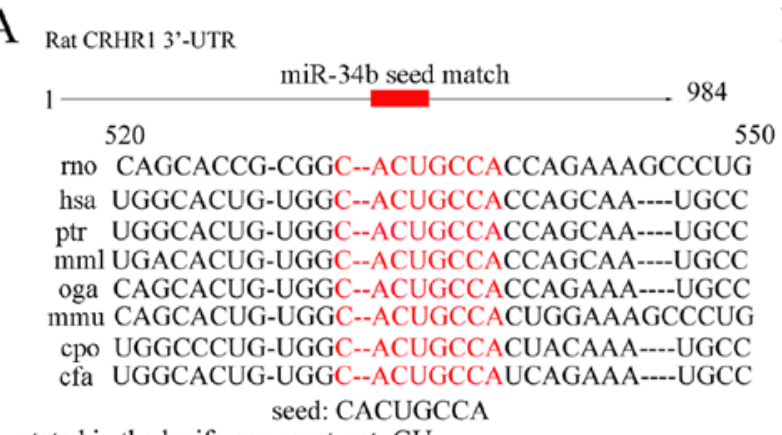

mutated in the luciferase construct: GU

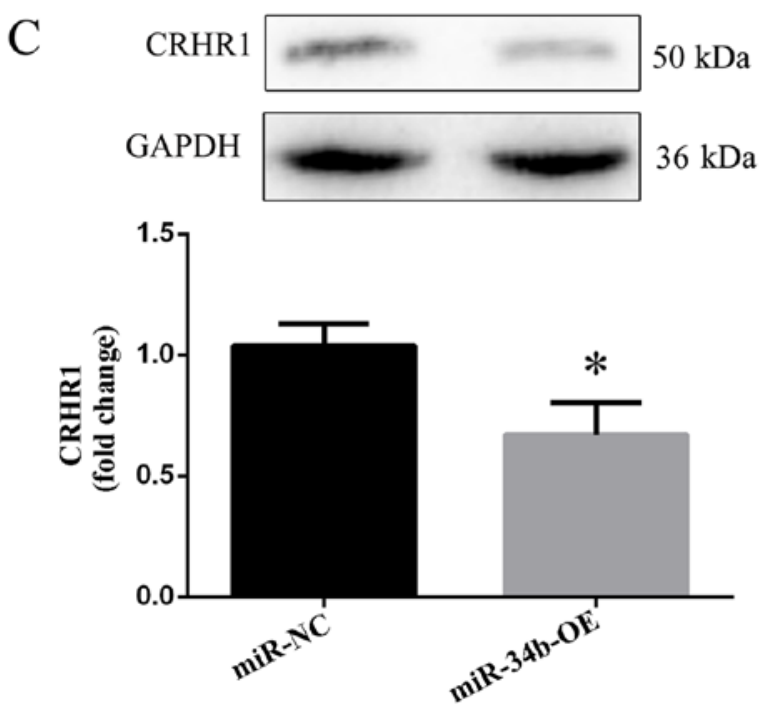

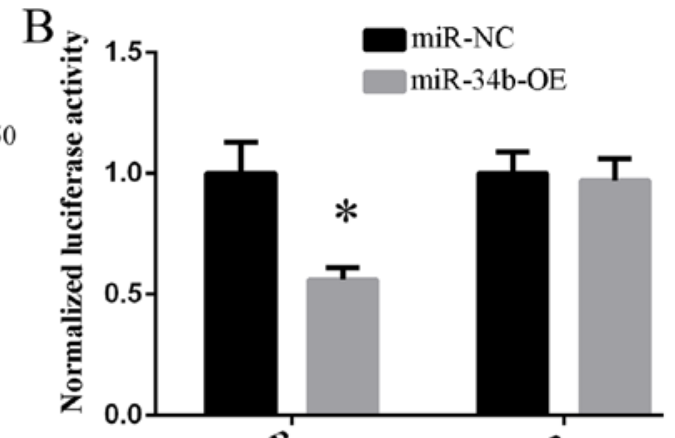
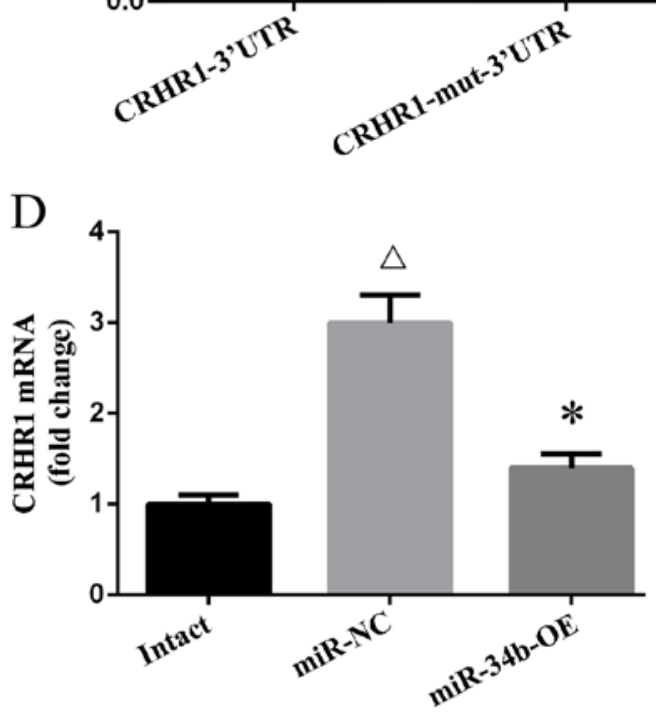

Forskolin $(10 \mu \mathrm{m})$

Figure 5. miR-34b modulates corticotropin-releasing hormone receptor 1 (CRHR1) mRNA level in primary cultured hypothalamus neurons. (A) Analysis of miR-34b binding site on the 3'-untranslated regions (3'-UTR) of CRHR1 mRNA. (B) Dual-Luciferase assays were performed in 293T cells. (C) Representative western blots of CRHR1 are shown with quantitative data after the overexpression treatment with miR-34b in primary cultured hypothalamus neurons. (D) Expression of CRHR1 mRNA after treatment with forskolin and miR-34b agomir in primary cultured hypothalamic neurons; $n=4$ for each group. " $\mathrm{p}<0.05$ represents statistically significant difference compared with the miRNA-NC group; ${ }^{\wedge} \mathrm{p}<0.05$ represents a statistically significant difference compared with the intact group.

bloodstream from the anterior pituitary gland where axons from parvocellular neurons in the PVN project through the median eminence. ACTH stimulates the synthesis and secretion of cortisol from the adrenal glands. Glucocorticoids can suppress $\mathrm{CRH}$ or ACTH gene expression in the hypothalamus or pituitary gland. It has been shown that the HPA axis modulates childhood trauma in the development of borderline personality disorder (17). The HPA axis has been shown to be associated with anxiety (18).

In the present study, a partial hepatectomy was used as a type of stress with which to excessively activate the HPA axis, as research has demonstrated an evident increase in CORT levels in rodents following a hepatectomy $(19,20)$ in order to facilitate liver regeneration. We found that the rats subjected to hepatectomy were anxious and experienced a dysfunction of the HPA axis, based upon the results of the OFT, EPM test and RIA.

As the CRH system in the hypothalamus is the central integrator in the HPA axis (21), a dysregulated CRH/CRHR1 system is considered to be one of the most common mechanisms that is associated with emotional disorders (22). CRHR1 exaggerates the $\mathrm{CRH}$ secretion in the hypothalamus as there is a positive ultrashort loop feedback of the CRH system in the hypothalamus (23). CRHR1 in the PVN has been shown to be involved in prenatal hypoxia exposure-induced anxiety in adult male rat offspring (24). CRHR1 in the amygdala has been reported to be involved in anxiety induced by environmental factors (25). The CRHR1 gene determines stress vulnerability (26) and contributes significantly to the depression severity rating (27). Another study demonstrated that the methylation of CRHR1 in the hypothalamus is linked to anxiety-like behavior (28). With increases in CRH, ACTH and CORT levels, CRHR1 was increased both at the mRNA and protein levels following surgery in the present study. Furthermore, the rats also expressed anxiety-like behavior. NBI-27914 is a CRHR1 specific antagonist $(29,30)$. In this study, we found that the application of NBI-27914 in the hypothalamus decreased the hyperactivity of the HPA axis and thus attenuated anxiety-like behavior by the inhibition of CRHR1. These results indicate that CRHR1 is involved in surgery-induced hyperactivity and anxiety.

miRNAs are small, non-coding RNAs that post-transcriptionally regulate gene expression. Hundreds of miRNAs have been identified throughout the brain and are involved in the regulation of neuronal development, function and dysfunc- 
A
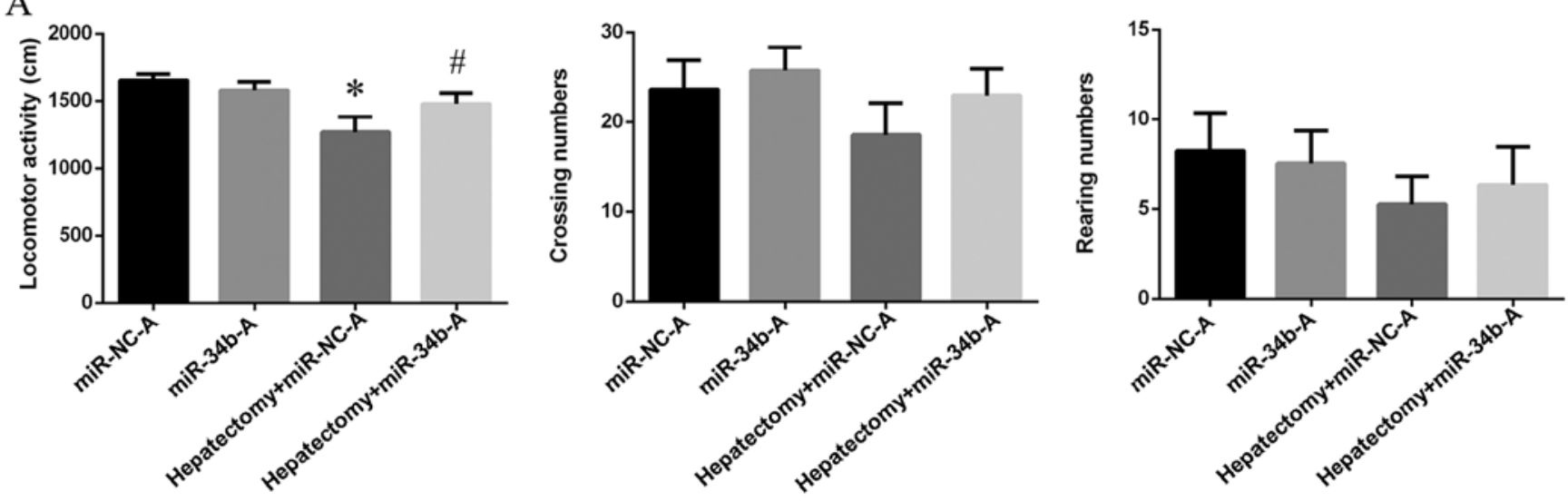

$\mathrm{B}$
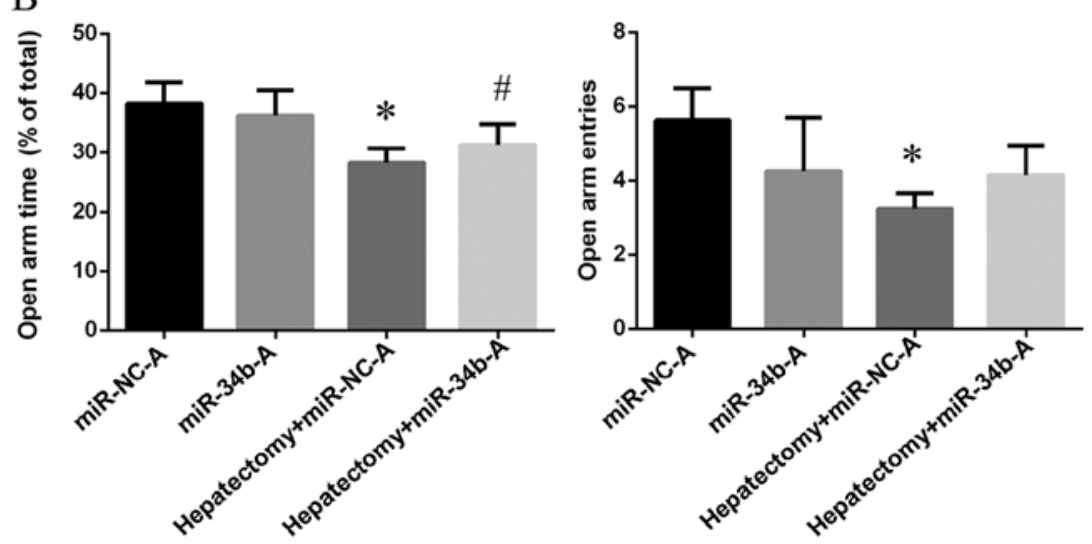

$\mathrm{C}$
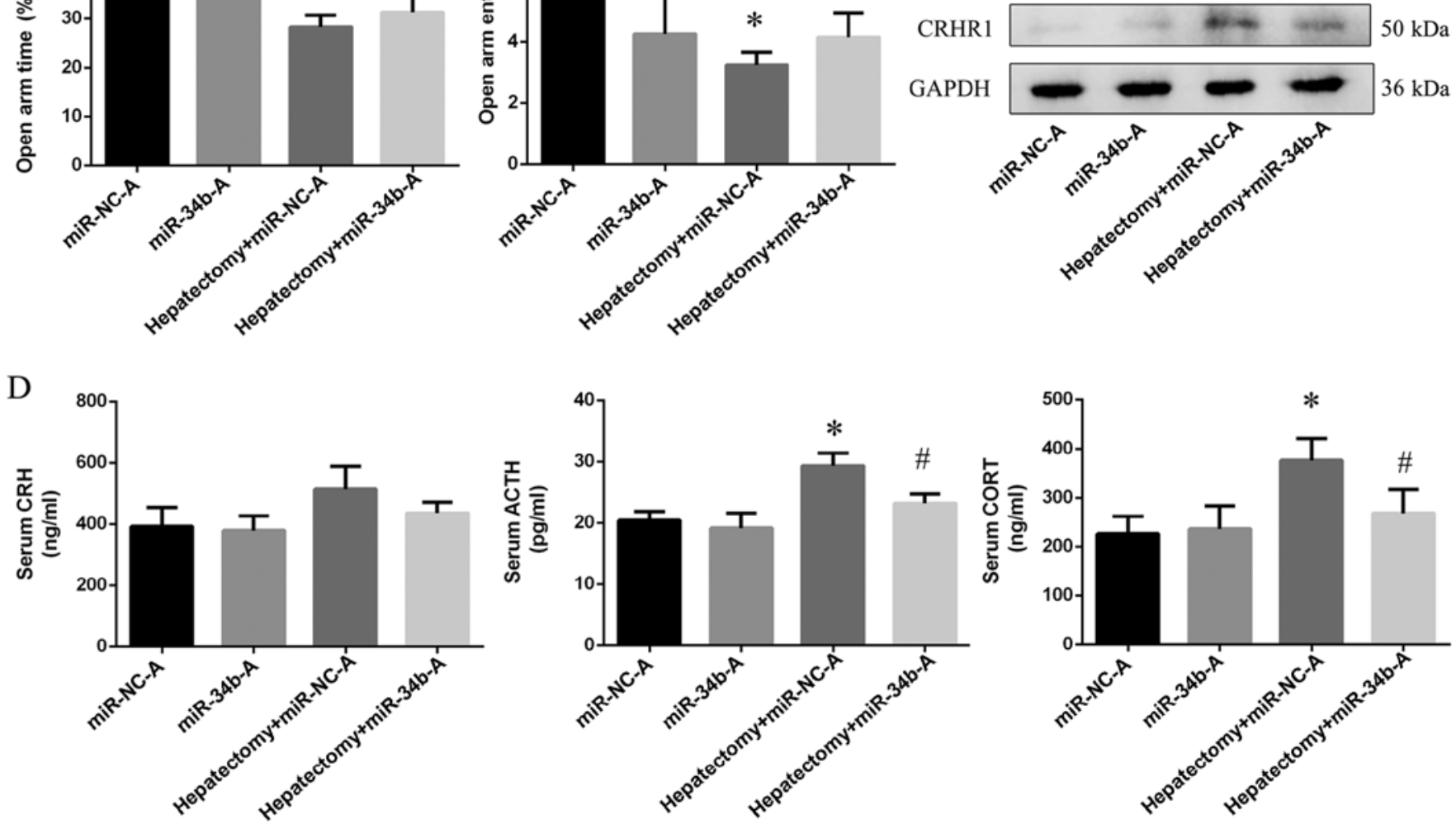

Figure 6. Surgery-induced anxiety and hyperactivity of the hypothalamic pituitary adrenal (HPA) axis was attenuated following treatment with miR-34b agomir. (A) Rat performance in open field test. (B) Rat performance in elevated plus maze test. (C) Representative western blots of the hypothalamus corticotropin-releasing hormone receptor 1 (CRHR1) are shown after miR-34b overexpression. (D) CRH, adrenocorticotropic hormone (ACTH) and CORT levels in serum. $\mathrm{n}=4$ for each group. ${ }^{*} \mathrm{p}<0.05$ represents statistically significant differences compared with the miR-NC-A group; ${ }^{*} \mathrm{p}<0.05$ represents statistically significant differences compared with the hepatectomy + miR-34b-A group.

tion (31). miRNAs have been proven to affect anxiety by altering synaptic plasticity (32). Serum levels of miRNA-132 and miRNA-128 have been reported to affect anxiety by targeting BDNF (33-35), which has been proven to mediate anxiety through neuronal neurogenesis and differentiation. The results from a previous study demonstrated that miR-134 mediate homeostatic synaptic depression (36).

miR-1202 enriched in the human brain has been shown to be associated with the pathophysiology of depression by targeting metabotropic glutamate receptor 4 (37). miR-185 and miR-491 also participate in the pathogenesis of depression (38).
Furthermore, miRNAs that possess polymorphisms may affect depression risk and treatment (39).

Nevertheless, limited research has been conducted concerning the association between miRNAs and the HPA axis in anxiety, particularly surgery-induced anxiety. The amygdala, hippocampus and other limbic structures can affect the function of the HPA axis (40).

Following bioinformatic analysis using TargetScan and miRWalk, 11 miRNAs were found to be overexpressed in primary hypothalamic neurons. miR-34b and miR-27a were found to have a negative association with CRHR1 mRNA. 
Forskolin was used to increase the expression of cyclic AMP in vitro and activated the $\mathrm{CRH}$ system, which has been proven to do so by previous studies $(41,42)$. In this study, we found an evident increase in activity in the CRH and AVP systems in primary hypothalamic neurons following treatment with forskolin. Moreover, accompanying this increasing trend in CRHR1 mRNA levels in the hypothalamic neurons, miR-34b was decreased following treatment with forskolin. Moreover, the overexpression of miR-34b decreased the CRHR1 protein levels and the forskolin-induced increase in the CRHR1 mRNA levels. A dual luciferase assay verified that miR-34b decreased the CRHR1 mRNA level by binding to its 3'-UTR. The overexpression of miR-34b by a miR-34b agomir that was injected into the PVN decreased the mRNA and protein level of CRHR1 in the hypothalamus and attenuated the dysfunction of the HPA axis and anxiety-like behavior.

In brief, the results of this study demonstrated that the HPA axis was hyperactive in rats following surgery and anxietylike behavior increased when compared with the intact (not operated) rats. We characterized the role of the HPA axis and miR-34b in the pathogenesis of trauma-induced anxiety and validated that miR-34b is a novel miRNA that regulates the CRHR1 mRNA level in the hypothalamus. We clarified that CRHR1 and its related miRNAs play an important role in the regulation of the HPA axis.

\section{Acknowledgements}

This study was supported by the National Key Program of Basic Science (973) 2013CB531906 and the National Natural Science Fund of China $(81573712,81471370)$.

\section{References}

1. Hossain M, Zimmerman C, Abas M, Light M and Watts C: The relationship of trauma to mental disorders among trafficked and sexually exploited girls and women. Am J Public Health 100 2442-2449, 2010.

2. Tully PJ, Winefield HR, Baker RA, Denollet J, Pedersen SS, Wittert GA and Turnbull DA: Depression, anxiety and major adverse cardiovascular and cerebrovascular events in patients following coronary artery bypass graft surgery: A five year longitudinal cohort study. Biopsychosoc Med 9: 14, 2015.

3. Castillo RC, Wegener ST, Heins SE, Haythornthwaite JA, Mackenzie EJ and Bosse MJ; LEAP Study Group: Longitudinal relationships between anxiety, depression, and pain: Results from a two-year cohort study of lower extremity trauma patients. Pain 154: 2860-2866, 2013.

4. Karanci AN and Dirik G: Predictors of pre- and postoperative anxiety in emergency surgery patients. J Psychosom Res 55: 363-369, 2003

5. Norrholm SD and Ressler KJ: Genetics of anxiety and traumarelated disorders. Neuroscience 164: 272-287, 2009.

6. Renoir T, Hasebe K and Gray L: Mind and body: How the health of the body impacts on neuropsychiatry. Front Pharmacol 4: 158, 2013.

7. Seitz DP, Bell CM, Gill SS, Reimer CL, Herrmann N, Anderson GM, Newman A and Rochon PA: Risk of perioperative blood transfusions and postoperative complications associated with serotonergic antidepressants in older adults undergoing hip fracture surgery. J Clin Psychopharmacol 33: 790-798, 2013.

8. Jeong BO, Kim SW, Kim SY, Kim JM, Shin IS and Yoon JS: Use of serotonergic antidepressants and bleeding risk in patients undergoing surgery. Psychosomatics 55: 213-220, 2014.

9. Pariante CM and Lightman SL: The HPA axis in major depression: Classical theories and new developments. Trends Neurosci 31: 464-468, 2008.
10. Zuloaga DG, Jacobskind JS and Raber J: Methamphetamine and the hypothalamic-pituitary-adrenal axis. Front Neurosci 9: 178, 2015.

11. Jacobson L: Hypothalamic-pituitary-adrenocortical axis: Neuropsychiatric aspects. Compr Physiol 4: 715-738, 2014.

12. Fan HM, Sun XY, Guo W, Zhong AF, Niu W, Zhao L, Dai YH, Guo ZM, Zhang LY and Lu J: Differential expression of microRNA in peripheral blood mononuclear cells as specific biomarker for major depressive disorder patients. J Psychiatr Res 59: 45-52, 2014

13. Durairaj RV and Koilmani ER: Environmental enrichment modulates glucocorticoid receptor expression and reduces anxiety in Indian field male mouse Mus booduga through upregulation of microRNA-124a. Gen Comp Endocrinol 199: 26-32, 2014.

14. Hanin G, Shenhar-Tsarfaty S, Yayon N, Yau YH, Bennett ER, Sklan EH, Rao DC, Rankinen T, Bouchard C, Geifman-Shochat S, et al: Competing targets of microRNA-608 affect anxiety and hypertension. Hum Mol Genet 23: 4569-4580, 2014.

15. Huang YN, Lai CC, Chiu CT, Lin JJ and Wang JY: L-ascorbate attenuates the endotoxin-induced production of inflammatory mediators by inhibiting MAPK activation and NF- $\kappa \mathrm{B}$ translocation in cortical neurons/glia Cocultures. PLoS One 9: e97276, 2014.

16. Sotnikov S, Wittmann A,Bunck M,Bauer S,Deussing J,SchmidtM, Touma C, Landgraf $\mathrm{R}$ and Czibere L: Blunted HPA axis reactivity reveals glucocorticoid system dysbalance in a mouse model of high anxiety-related behavior. Psychoneuroendocrinology 48: 41-51, 2014.

17. Martín-Blanco A, Ferrer M, Soler J, Arranz MJ, Vega D, Calvo N, Elices M, Sanchez-Mora C, García-Martinez I, Salazar J, et al: The role of hypothalamus-pituitary-adrenal genes and childhood trauma in borderline personality disorder. Eur Arch Psychiatry Clin Neurosci 266: 307-316, 2016.

18. Li C, Liu Y, Yin S, Lu C, Liu D, Jiang H and Pan F: Long-term effects of early adolescent stress: Dysregulation of hypothalamicpituitary-adrenal axis and central corticotropin releasing factor receptor 1 expression in adult male rats. Behav Brain Res 288: 39-49, 2015.

19. Cipriano C, Giacconi R, Muzzioli M, Gasparini N, Orlando F, Corradi A, Cabassi E and Mocchegiani E: Metallothionein (I+II) confers, via c-myc, immune plasticity in oldest mice: Model of partial hepatectomy/liver regeneration. Mech Ageing Dev 124: 877-886, 2003.

20. Witek-Janusek L, Yu M and Marotta SF: Hypoxic and nycthemeral responses by the adrenal cortex of partially hepatectomized rats. Aviat Space Environ Med 55: 538-541, 1984.

21. Bonfiglio JJ, Inda C, Refojo D, Holsboer F, Arzt E and Silberstein S: The corticotropin-releasing hormone network and the hypothalamic-pituitary-adrenal axis: Molecular and cellular mechanisms involved. Neuroendocrinology 94: 12-20, 2011.

22. Wasserman D, Wasserman J and Sokolowski M: Genetics of HPA-axis, depression and suicidality. Eur Psychiatry 25: 278-280, 2010.

23. Ono N, Samson WK, McDonald JK, Lumpkin MD, Bedran de Castro JC and McCann SM: Effects of intravenous and intraventricular injection of antisera directed against corticotropin-releasing factor on the secretion of anterior pituitary hormones. Proc Natl Acad Sci USA 82: 7787-7790, 1985.

24. Fan JM, Wang X, Hao K, Yuan Y, Chen XQ and Du JZ: Upregulation of PVN CRHR1 by gestational intermittent hypoxia selectively triggers a male-specific anxiogenic effect in rat offspring. Horm Behav 63: 25-31, 2013.

25. Sotnikov SV, Chekmareva NY, Schmid B, Harbich D, Malik V, Bauer S, Kuehne C, Markt PO, Deussing JM, Schmidt MV, et al: Enriched environment impacts trimethylthiazoline-induced anxiety-related behavior and immediate early gene expression: Critical role of Crhr1. Eur J Neurosci 40: 2691-2700, 2014.

26. Labermaier C, Kohl C, Hartmann J, Devigny C, Altmann A, Weber P, Arloth J, Quast C, Wagner KV, Scharf SH, et al: A polymorphism in the Crhrl gene determines stress vulnerability in male mice. Endocrinology 155: 2500-2510, 2014.

27. Schatzberg AF, Keller J, Tennakoon L, Lembke A, Williams G, Kraemer FB, Sarginson JE, Lazzeroni LC and Murphy GM: HPA axis genetic variation, cortisol and psychosis in major depression. Mol Psychiatry 19: 220-227, 2014.

28. Wang X, Meng FS, Liu ZY, Fan JM, Hao K, Chen XQ and Du JZ: Gestational hypoxia induces sex-differential methylation of Crhr1 linked to anxiety-like behavior. Mol Neurobiol 48: 544-555, 2013. 
29. Silberman Y and Winder DG: Corticotropin releasing factor and catecholamines enhance glutamatergic neurotransmission in the lateral subdivision of the central amygdala. Neuropharmacology 70: 316-323, 2013.

30. Lowery-Gionta EG, Navarro M, Li C, Pleil KE, Rinker JA, Cox BR, Sprow GM, Kash TL and Thiele TE: Corticotropin releasing factor signaling in the central amygdala is recruited during binge-like ethanol consumption in C57BL/6J mice. J Neurosci 32: 3405-3413, 2012.

31. Im HI and Kenny PJ: MicroRNAs in neuronal function and dysfunction. Trends Neurosci 35: 325-334, 2012.

32. Hu Z, Yu D, Gu QH, Yang Y, Tu K, Zhu J and Li Z: miR-191 and miR-135 are required for long-lasting spine remodelling associated with synaptic long-term depression. Nat Commun 5 : 3263, 2014

33. Li YJ, Xu M, Gao ZH, Wang YQ, Yue Z, Zhang YX, Li XX, Zhang C, Xie SY and Wang PY: Alterations of serum levels of BDNF-related miRNAs in patients with depression. PLoS One 8: e63648, 2013.

34. Briones TL and Woods J: Chronic binge-like alcohol consumption in adolescence causes depression-like symptoms possibly mediated by the effects of BDNF on neurogenesis. Neuroscience 254: 324-334, 2013

35. Yoneyama M, Tanaka M, Hasebe S, Yamaguchi T, Shiba T and Ogita K: Beneficial effect of cilostazol-mediated neuronal repair following trimethyltin-induced neuronal loss in the dentate gyrus. J Neurosci Res 93: 56-66, 2015.

36. Fiore R, Rajman M, Schwale C, Bicker S, Antoniou A, Bruehl C, Draguhn A and Schratt G: MiR-134-dependent regulation of Pumilio-2 is necessary for homeostatic synaptic depression. EMBO J 33: 2231-2246, 2014.
37. Lopez JP, Lim R, Cruceanu C, Crapper L, Fasano C, Labonte B, Maussion G, Yang JP, Yerko V, Vigneault E, et al: miR-1202 is a primate-specific and brain-enriched microRNA involved in major depression and antidepressant treatment. Nat Med 20: 764-768, 2014.

38. Serafini G, Pompili M, Hansen KF, Obrietan K, Dwivedi Y, Shomron N and Girardi P: The involvement of microRNAs in major depression, suicidal behavior, and related disorders: A focus on miR-185 and miR-491-3p. Cell Mol Neurobiol 34: 17-30, 2014.

39. He Y, Zhou Y, Xi Q, Cui H, Luo T, Song H, Nie X, Wang L and Ying B: Genetic variations in microRNA processing genes are associated with susceptibility in depression. DNA Cell Biol 31: 1499-1506, 2012.

40. Meyer DL, Davies DR, Barr JL, Manzerra P and Forster GL: Mild traumatic brain injury in the rat alters neuronal number in the limbic system and increases conditioned fear and anxiety-like behaviors. Exp Neurol 235: 574-587, 2012.

41. Kageyama K, Itoi K, Iwasaki Y, Niioka K, Watanuki Y, Yamagata S, Nakada Y, Das G, Suda T and Daimon M: Stimulation of corticotropin-releasing factor gene expression by FosB in rat hypothalamic 4B cells. Peptides 51: 59-64, 2014.

42. Nikodemova M, Kasckow J, Liu H, Manganiello V and Aguilera G: Cyclic adenosine 3',5'-monophosphate regulation of corticotropin-releasing hormone promoter activity in AtT-20 cells and in a transformed hypothalamic cell line. Endocrinology 144: 1292-1300, 2003 